\title{
ESSENTIAL m-SECTORIALITY AND ESSENTIAL SPECTRUM OF THE SCHRÖDINGER OPERATORS WITH RAPIDLY OSCILLATING COMPLEX-VALUED POTENTIALS
}

\author{
By
}

\author{
Yorimasa Oshime
}

\begin{abstract}
Schrödinger operators $T_{0}=-\Delta+q(x)$ with rapidly oscillating complex-valued potentials $q(x)$ are considered. Each of such operators is sectorial and hence has Friedrichs extension. We prove that $T_{0}$ is essentially m-sectorial in the sense that the closure of $T_{0}$ coincides with its Friedrichs extension $T$. In particular, $T_{0}$ is essentially self-adjoint if the rapidly oscillating potential $q(x)$ is realvalued. Further, we prove $\sigma_{\text {ess }}(T)=[0, \infty)$ under somewhat stricter condition on the potentials $q(x)$.
\end{abstract}

\section{Introduction}

It is well known (see Theorem X.38 and its corollary in Reed-Simon [4]) that the Schrödinger operator $-\triangle+q(x)\left(x \in \mathbf{R}^{N}\right)$ is essentially self-adjoint if the real potential $q(x)$ satisfies $q(x) \geq-c|x|^{2}$ for some positive constant $c$. However, there are still many potentials for which the essential self-adjointness of the Schrödinger operators have not been fully studied. Rapidly oscillating potentials are among such ones and typical examples are

$$
\varphi\left(\frac{x}{|x|}\right)|x|^{3} \sin \left(|x|^{5}\right),\left(1+|x|^{2}\right)^{-1} e^{|x|} \cos \left(e^{|x|}\right) .
$$

Here $\varphi(\omega)$ is a bounded function on the unit sphere $S^{N-1}=\left\{\omega \in \mathbf{R}^{N}:|\omega|=1\right\}$. Skriganov [6] (see also Mateev and Skriganov [3]) studies such potentials and also

2010 Mathematics Subject Classification: Primary 35J10; Secondary 35P15.

Key words and phrases: Oscillating potentials, Sectorial forms, Friedrichs extension.

Received September 10, 2014.

Revised June 30, 2015. 
provides sufficient conditions for the essential self-adjointness of the operators. However, he assumes that the potentials are continuous and satisfy some additional properties. Removing the continuity conditions on the potentials, Sasaki [5] proves that the essential spectrum of their Friedrichs extension is $[0, \infty)$ though he does not consider their essential self-adjointness.

It should be noted that the above authors use argument applicable only to the real potentials. In this paper, we study complex-valued rapidly oscillating potentials. To mention our results, we define the essential m-sectoriality of operators.

Let $S_{0}$ be a densely defined sectorial operator in a Hilbert space. Then $S_{0}$ has an m-sectorial extension $S$ which is called its Friedrichs extension. (See Kato [2, p325].) If this $S$ coincides with the closure of $S_{0}$, then $S_{0}$ is called essentially $m$-sectorial. In the special case where $S_{0}$ is a symmetric operator bounded from below, the essential m-sectoriality becomes the essential self-adjointness.

In Section 2, we prove the essential self-adjointness or rather the essential $\mathrm{m}$-sectoriality of the operators with complex-valued rapidly oscillating potentials, avoiding continuity conditions. It is guaranteed that, for example, $T_{0}=-\triangle+q(x)$, $\operatorname{Dom}\left(T_{0}\right)=C_{0}^{\infty}$ with $q(x)=|x|^{3} e^{i|x|^{4}}$ or $e^{|x|} \exp \left(i e^{|x|}\right)$ is essentially m-sectorial and its closure coincides with its Friedrichs extension.

In Section 3, we prove that the essential spectrum of such operators equals $[0, \infty)$ under somewhat stricter condition on the potentials. It is guaranteed that, for example, the Friedrichs extension $T$ of $T_{0}=-\triangle+q(x)$ with $q(x)=|x|^{3} e^{i|x|^{5}}$ or $\left(1+|x|^{2}\right)^{-1} e^{|x|} \exp \left(i e^{|x|}\right)$ satisfies $\sigma_{\text {ess }}(T)=[0, \infty)$.

Our main tools are sectorial sesquilinear forms and associated m-sectorial operators. See Kato [2] for their definitions and basic properties.

Finally, the author sincerely thanks Professor I. Sasaki of Shinshu University for many valuable advices and encouragement. He also thanks the referee for many suggestions to improve the manuscript.

\section{Essential m-Sectoriality}

In this section, we consider the essential m-sectoriality of the operator

$$
T_{0} u=-\triangle u+q(x) u, \quad\left(x \in \mathbf{R}^{N}\right)
$$

with domain $\operatorname{Dom}\left(T_{0}\right)=C_{0}^{\infty}\left(\mathbf{R}^{N}\right)$.

Throughout this section, we always assume

$$
q(x)=q_{1}(x)+q_{2}(x)
$$


with $q_{1}(x) \in L_{l o c}^{\infty}\left(\mathbf{R}^{N}\right), q_{2}(x) \in L^{\infty}\left(\mathbf{R}^{N}\right)$ and

$$
\sup _{r>0, \omega \in S^{N-1}}\left|\int_{0}^{r} q_{1}(\rho \omega) d \rho\right|<\infty .
$$

Therefore, by setting

$$
Q_{1}(r \omega)=\int_{0}^{r} q_{1}(\rho \omega) d \rho,
$$

$q_{1}(x) \in L_{l o c}^{\infty}\left(\mathbf{R}^{N}\right)$ implies

$$
\begin{array}{r}
\left|Q_{1}(r \omega)\right| \leq M \min \{1, r\}, \\
\sup _{r>0, \omega \in S^{N-1}}\left|q_{2}(r \omega)\right| \leq M
\end{array}
$$

for some constant $M>0$ independent of $\omega \in S^{N-1}$.

Note that $q(x)=|x|^{3} e^{i|x|^{4}}$ are $e^{|x|} \exp \left(i e^{|x|}\right)$ typical examples for the above $q_{1}(x)$.

Lemma 1. For $u \in H^{1}\left(\mathbf{R}^{N}\right), v \in C_{0}^{\infty}\left(\mathbf{R}^{N}\right)$,

$$
\begin{aligned}
\int_{\mathbf{R}^{N}} q_{1}(x) u(x) \overline{v(x)} d x= & -(N-1) \int_{\mathbf{R}^{N}}\left(Q_{1}(x) /|x|\right) u(x) \overline{v(x)} d x \\
& -\int_{\mathbf{R}^{N}} Q_{1}(x) \sum_{j=1}^{N}\left(x_{j} /|x|\right)\left\{u\left(\partial \bar{v} / \partial x_{j}\right)+\left\{\left(\partial u / \partial x_{j}\right) \bar{v}\right\} d x .\right.
\end{aligned}
$$

Proof. We may assume $u \in C_{0}^{\infty}\left(\mathbf{R}^{N}\right)$. Using $(\partial / \partial r) Q_{1}(r \omega)=q_{1}(r \omega)$ and integration by parts, we have

$$
\begin{aligned}
\int_{\mathbf{R}^{N}} q_{1}(x) u(x) \overline{v(x)} d x= & \int_{S^{N-1}} \int_{0}^{\infty} r^{N-1} q_{1}(r \omega) u(r \omega) \overline{v(r \omega)} d r d \omega \\
= & -(N-1) \int_{\mathbf{R}^{N}}\left(Q_{1}(x) /|x|\right) u(x) \overline{v(x)} d x \\
& -\int_{\mathbf{R}^{N}} Q_{1}(x) \sum_{j=1}^{N}\left(x_{j} /|x|\right)\left\{u\left(\partial \bar{v} / \partial x_{j}\right)+\left(\partial u / \partial x_{j}\right) \bar{v}\right\} d x
\end{aligned}
$$


Lemma 2. Define the sesqulinear form $s[u, v]$ by

$$
\begin{aligned}
s[u, v]= & -(N-1) \int_{\mathbf{R}^{N}} \frac{Q_{1}(x)}{|x|} u(x) \overline{v(x)} d x \\
& -\int_{\mathbf{R}^{N}} Q_{1}(x) \sum_{j=1}^{N} \frac{x_{j}}{|x|}\left\{\frac{\partial u(x)}{\partial x_{j}} \overline{v(x)}+u(x) \frac{\overline{\partial v(x)}}{\partial x_{j}}\right\} d x \\
& +\int_{\mathbf{R}^{N}} q_{2}(x) u(x) \overline{v(x)} d x
\end{aligned}
$$

for $u, v \in H^{1}\left(\mathbf{R}^{N}\right)$. Then we have

$$
|s[u, v]| \leq M\|\nabla u\|_{L^{2}}\|v\|_{L^{2}}+M\|u\|_{L^{2}}\|\nabla v\|_{L^{2}}+M N\|u\|_{L^{2}}\|v\|_{L^{2}}
$$

for all $u, v \in H^{1}\left(\mathbf{R}^{N}\right)$.

Proof. Recalling $\left|Q_{1}(x)\right| \leq M$ and using the Cauchy-Schwartz inequality, we have

$$
\left|\int_{\mathbf{R}^{N}} Q_{1}(x) \sum_{j=1}^{N} \frac{x_{j}}{|x|}\left\{\frac{\partial u(x)}{\partial x_{j}} \overline{v(x)}+u(x) \frac{\overline{\partial v(x)}}{\partial x_{j}}\right\} d x\right| \leq M\|\nabla u\|\|v\|+M\|u\|\|\nabla v\| .
$$

We apply $\left|Q_{1}(x)\right| \leq M|x|$ and $\left|q_{2}(x)\right| \leq M$ to the first and third terms of $s[u, v]$ to obtain

$$
|s[u, v]| \leq M\|\nabla u\|\|v\|+M\|u\|\|\nabla v\|+M N\|u\|\|v\|
$$

Remark. Combining Lemma 1 and Lemma 2 we know that the multiplication operator $u \rightarrow\left(q_{1}(x)+q_{2}(x)\right) u$ can be extended to a bounded map from $H^{1}$ to $H^{-1}$.

THEOREM 3.

$$
t[u, v]=(\nabla u, \nabla v)+s[u, v]
$$

is a closed sectorial sesquilinear form with domain $H^{1}$. The associated m-sectorial operator is

$$
T u=-\triangle u+q(x) u
$$

with domain

$$
\operatorname{Dom}(T)=\left\{u \in H^{1} \cap H_{l o c}^{2}:-\triangle u+q(x) u \in L^{2}\right\}
$$


Proof. From the previous Lemma 2, we have

$$
|s[u, u]| \leq(1 / 2)\|\nabla u\|^{2}+\left(8 M^{2}+M N\right)\|u\|^{2} .
$$

Hence

$$
\operatorname{Re} t[u, u]=\|\nabla u\|^{2}+\operatorname{Re} s[u, u] \geq(1 / 2)\|\nabla u\|^{2}-\left(8 M^{2}+M N\right)\|u\|^{2} .
$$

We also have

$$
|t[u, v]| \leq\|\nabla u\|\|\nabla v\|+M\|\nabla u\|\|v\|+M\|u\|\|\nabla v\|+M N\|u\|\|v\| .
$$

Therefore $t[u, v]$ is a closed sectorial sesquilinear form with domain $H^{1}$. Thus the representation theorem (Theorem 2.1 of Kato, [2; p322]) ensures that there exists a unique associated $\mathrm{m}$-sectorial operator $T$ such that for an arbitrary $u \in \operatorname{Dom}(T), t[u, v]=(T u, v)$ holds for all $v \in \operatorname{Dom}(t)$.

Finally, considering the special case where $v \in C_{0}^{\infty}$, it is easy to prove $\operatorname{Dom}(T)=\left\{u \in H^{1} \cap H_{l o c}^{2}:-\triangle u+q(x) u \in L^{2}\right\}$.

We have now proved the minimal operator $T_{0} u=-\triangle u+q(x) u$ with domain $\operatorname{Dom}\left(T_{0}\right)=C_{0}^{\infty}$ has an m-sectorial extension (i.e., Friedrichs extension). We shall show this extension is unique by proving the closure of $T_{0}$ is exaclty the Friedrichs extension $T$ we have just obtained. Let us begin with a lemma.

Lemma 4. For any $u \in H_{l o c}^{1}$ and any constant $R \geq 1$, the following holds.

$$
\left.\left.\left|\int_{|x| \leq R} q_{1}(x)\right| u(x)\right|^{2} d x\left|\leq(1 / 2) \int_{|x| \leq R}\right| \nabla u\right|^{2} d x+\left(8 M^{2}+2 M N\right) \int_{|x| \leq R}|u|^{2} d x .
$$

Proof. We may assume $u \in H_{l o c}^{1} \cap C^{\infty}$. Note that

$$
\begin{aligned}
\int_{|x| \leq R} q_{1}(x)|u(x)|^{2} d x= & \int_{S^{N-1}} Q_{1}(R \omega) R^{N-1}|u(R \omega)|^{2} d \omega \\
& -\int_{S^{N-1}} \int_{0}^{R}(N-1) r^{N-2} Q_{1}(r \omega)|u(r \omega)|^{2} d r d \omega \\
& -\int_{S^{N-1}} \int_{0}^{R} r^{N-1} Q_{1}(r \omega) \frac{\partial}{\partial r}|u(r \omega)|^{2} d r d \omega .
\end{aligned}
$$


Recalling $\left|Q_{1}(r \omega)\right| \leq M \min \{r, 1\}$, we further have

$$
\begin{aligned}
\left.\left|\int_{|x| \leq R} q_{1}(x)\right| u(x)\right|^{2} d x \mid \leq & M \int_{S^{N-1}} R^{N-1}|u(R \omega)|^{2} d \omega+M(N-1) \int_{|x| \leq R}|u(x)|^{2} d x \\
& +2 M \int_{|x| \leq R}|u(x)||\nabla u(x)| d x \\
\leq & M \int_{S^{N-1}} R^{N-1}|u(R \omega)|^{2} d \omega+(1 / 4) \int_{|x| \leq R}|\nabla u(x)|^{2} d x \\
& +\left(4 M^{2}+M N\right) \int_{|x| \leq R}|u(x)|^{2} d x .
\end{aligned}
$$

Now we have only to estimate $\int_{S^{N-1}} R^{N-1}|u(R \omega)|^{2} d \omega$. Indeed,

$$
\begin{array}{rl}
\int_{S^{N-1}} & R^{N-1}|u(R \omega)|^{2} d \omega \\
= & \int_{S^{N-1}} \int_{1}^{R} \frac{\partial}{\partial r} r^{N-1}|u(r \omega)|^{2} d r d \omega+\int_{S^{N-1}} \int_{0}^{1} \frac{\partial}{\partial r} r^{N}|u(r \omega)|^{2} d r d \omega \\
= & \int_{S^{N-1}} \int_{1}^{R}(N-1) r^{N-2}|u(r \omega)|^{2} d r d \omega \\
& +\int_{S^{N-1}} \int_{1}^{R} 2 r^{N-1} \operatorname{Re} u(r \omega)(\omega \cdot \overline{\nabla u(r \omega)}) d r d \omega \\
& +\int_{S^{N-1}} \int_{0}^{1} N r^{N-1}|u(r \omega)|^{2} d r d \omega \\
& +\int_{S^{N-1}} \int_{0}^{1} 2 r^{N} \operatorname{Re} u(r \omega)(\omega \cdot \overline{\nabla u(r \omega)}) d r d \omega \\
\leq & \int_{1 \leq|x| \leq R}(N-1)|x|^{-1}|u(x)|^{2} d x+\int_{1 \leq \mid x \leq R} 2|u(x)||\nabla u| d x \\
& +\int_{|x| \leq 1} N|u(x)|^{2} d x+\int_{|x| \leq 1} 2|u(x)||\nabla u| d x \\
\leq & N \int_{|x| \leq R}|u(x)|^{2} d x+2 \int_{|x| \leq R}|u(x)||\nabla u| d x \\
4 M & 1 \\
|x| \leq R & |\nabla u|^{2} d x+(N+4 M) \int_{|x| \leq R}|u(x)|^{2} d x . \\
\mathbf{\square}
\end{array}
$$


Proposition 5. Suppose $u \in L^{2}$ satisfies

$$
-\triangle u+(q(x)-\lambda) u=w \in L^{2} .
$$

in the distributional sense, for some complex constant $\lambda$. Then $u \in H^{1} \cap H_{\text {loc }}^{2}$.

Proof. First notice that

$$
-\triangle u=-(q(x)-\lambda) u+w \in L_{l o c}^{2}
$$

since $q(x)=q_{1}(x)+q_{2}(x)$ is locally bounded. Hence $u \in H_{l o c}^{2}$.

Observe now that

$$
\int_{|x| \leq R} \bar{u} \Delta u=-\int_{|x| \leq R}|\nabla u|^{2} d x+\int_{S^{N-1}} R^{N-1} \bar{u}(R \omega) \frac{\partial u}{\partial R}(R \omega) d \omega
$$

since $u \in H_{l o c}^{2}$. Note that the integral on $S^{N-1}$ converges. From this equation and $\triangle u=\left(q_{1}(x)+q_{2}(x)-\lambda\right) u-w$,

$$
\begin{aligned}
& -\int_{|x| \leq R}|\nabla u|^{2} d x+\int_{S^{N-1}} R^{N-1}|u(R \omega)||\nabla u(R \omega)| d \omega \\
& \quad \geq \operatorname{Re} \int_{|x| \leq R} \bar{u} \Delta u d x \\
& \quad=\operatorname{Re} \int_{|x| \leq R}\left\{\left(q_{1}(x)+q_{2}(x)-\lambda\right)|u(x)|^{2}-\overline{u(x)} w(x)\right\} d x \\
& \geq-\left.\left|\int_{|x| \leq R} q_{1}(x)\right| u(x)\right|^{2} d x \mid-\int_{|x| \leq R}\left\{\left|q_{2}(x)-\lambda\right||u(x)|^{2}+|u(x)||w(x)|\right\} d x
\end{aligned}
$$

By Lemma 4 and $\left|q_{2}(x)-\lambda\right| \leq M+|\lambda|$, this is estimated from below by

$$
-(1 / 2) \int_{|x| \leq R}|\nabla u|^{2} d x-\left(8 M^{2}+2 M N+M+|\lambda|\right)\|u\|^{2}-\|u\|\|w\| .
$$

Therefore, we have

$$
\begin{aligned}
& (1 / 2) \int_{|x| \leq R}|\nabla u|^{2} d x-\left(8 M^{2}+2 M N+M+|\lambda|\right)\|u\|^{2}-\|u\|\|w\| \\
& \quad \leq \int_{S^{N-1}} R^{N-1}|u(R \omega)||\nabla u(R \omega)| d \omega .
\end{aligned}
$$


Let us now prove $u \in H^{1}$ by contradiction, assuming to the contrary that

$$
\lim _{R \rightarrow \infty} \int_{|x| \leq R}|\nabla u|^{2} d x=\infty .
$$

Thus

$$
(1 / 4) \int_{|x| \leq R}|\nabla u|^{2} d x \leq \int_{S^{N-1}} R^{N-1}|u(R \omega)||\nabla u(R \omega)| d \omega
$$

for $R \geq R_{0}$ with sufficiently large $R_{0}$. Putting

$$
G(R)=\int_{|x| \leq R}|\nabla u|^{2} d x
$$

and integrating the last inequality over $\left[R_{0}, R\right]$, we have

$$
\begin{aligned}
(1 / 4) \int_{R_{0}}^{R} G(r) d r & \leq \int_{R_{0}}^{R} \int_{S^{N-1}} r^{N-1}|u(r \omega)||\nabla u(r \omega)| d \omega d r \\
& \leq \int_{R_{0} \leq|x| \leq R}|u(x)||\nabla u(x)| d x \\
& \leq\|u\|(G(R))^{1 / 2} .
\end{aligned}
$$

Hence we have

$$
\frac{\|u\|^{-2}}{16} \leq \frac{G(R)}{\left(\int_{R_{0}}^{R} G(r) d r\right)^{2}} \quad\left(R \geq R_{0}\right)
$$

Integrating this inequality over $\left[2 R_{0}, R\right]$, we have

$$
\frac{\left(R-2 R_{0}\right)\|u\|^{-2}}{16} \leq\left(\int_{R_{0}}^{2 R_{0}} G(r) d r\right)^{-1}-\left(\int_{R_{0}}^{R} G(r) d r\right)^{-1}<\infty .
$$

If $R \rightarrow \infty$, the left side goes to $\infty$ while the right side remains bounded.

A contradiction.

Now we are ready to prove the main theorem of this section.

THEOREM 6. Let

$$
T_{0} u=-\triangle u+q(x) u, \quad \operatorname{Dom}\left(T_{0}\right)=C_{0}^{\infty}\left(\mathbf{R}^{N}\right) .
$$


Assume

$$
q(x)=q_{1}(x)+q_{2}(x), \quad q_{1}(x) \in L_{l o c}^{\infty}\left(\mathbf{R}^{N}\right), \quad q_{2}(x) \in L^{\infty}\left(\mathbf{R}^{N}\right)
$$

and

$$
\sup _{r>0, \omega \in S^{N-1}}\left|\int_{0}^{r} q_{1}(\rho \omega) d \rho\right|<\infty .
$$

Then the closure $\overline{T_{0}}$ of $T_{0}$ coincides with its Friedrichs extension $T$.

Proof. Let $\lambda$ be outside the sectorial regions (which are larger than the numerical ranges) of the sesquilinear forms $t[u, v]$ and $t^{*}[u, v]=\overline{t[v, u]}$. Note that $\lambda \in \rho(T) \cap \rho\left(T^{*}\right)$. Suppose there exists $v \in L^{2} \backslash\{0\}$ such that

$$
\left(v,\left(T_{0}-\lambda\right) u\right)=0
$$

for all $u \in C_{0}^{\infty}$. This means $v \in L^{2}$ is the distributional solution of

$$
-\triangle v+\overline{(q(x)-\lambda)} v=0
$$

Therefore, the previous Proposition 5 implies that $v \in H^{1}=\operatorname{Dom}\left(t^{*}\right)=\operatorname{Dom}(t)$. Hence

$$
\left(t^{*}-\bar{\lambda}\right)[v, v]=\overline{(t-\lambda)[v, v]}=(-\Delta v+\overline{(q(x)-\lambda)} v, v)=0 .
$$

Recalling that $\lambda$ is outside the sectorial region of the sesquilinear form $t[u, v]$, we obtain $v=0$. Thus we have proved that

$$
\overline{\operatorname{Ran}\left(\overline{T_{0}}-\lambda\right)}=\overline{\operatorname{Ran}\left(T_{0}-\lambda\right)}=L^{2} .
$$

Since $\left(\overline{T_{0}}-\lambda\right) \subseteq(T-\lambda)$ and $(T-\lambda)^{-1}$ is bounded, so is $\left(\overline{T_{0}}-\lambda\right)^{-1}$ on its domain $\operatorname{Ran}\left(\overline{T_{0}}-\lambda\right)$. Recalling that $\overline{T_{0}}-\lambda$ is a closed operator, we obtain

$$
\operatorname{Ran}\left(\overline{T_{0}}-\lambda\right)=\overline{\operatorname{Ran}\left(\overline{T_{0}}-\lambda\right)}=L^{2}=\operatorname{Ran}(T-\lambda) .
$$

This implies $\left(\overline{T_{0}}-\lambda\right)=(T-\lambda)$ and $\overline{T_{0}}=T$.

REMARK. If, in addition, $q_{1}(x)$ and $q_{2}(x)$ in $q(x)=q_{1}(x)+q_{2}(x)$ are both real-valued, then Theorem 6 ensures that $T_{0} u=-\triangle u+q(x) u$ is essentially selfadjoint. 


\section{Essential Spectrum}

In this section, imposing somewhat stricter conditions on the potential $q(x)=$ $q_{1}(x)+q_{2}(x)$, we study the essential spectrum of the Friedrichs extension $T$ of $-\triangle+q(x)$. More specifically, we assume that $q_{1}(x) \in L_{l o c}^{\infty}\left(\mathbf{R}^{N}\right), q_{2}(x) \in L^{\infty}\left(\mathbf{R}^{N}\right)$ satisfy

$$
\lim _{r_{1}, r_{2} \rightarrow \infty} \sup _{\omega \in S^{N-1}}\left|\int_{r_{1}}^{r_{2}} q_{1}(\rho \omega) d \rho\right|=0
$$

and

$$
\lim _{r \rightarrow \infty} \sup _{\omega \in S^{N-1}}\left|q_{2}(r \omega)\right|=0
$$

throughout this section. In other words, for any $\omega \in S^{N-1}$ and $r_{2}>r_{1} \geq R$, we assume

$$
\left|Q_{1}\left(r_{2} \omega\right)-Q_{1}\left(r_{1} \omega\right)\right|+\left|q_{2}\left(r_{1} \omega\right)\right|<\varepsilon(R)
$$

with some $\varepsilon(R)$ such that $\lim _{R \rightarrow \infty} \varepsilon(R)=0$.

Note that $q(x)=|x|^{3} e^{i|x|^{5}}$ and $\left(1+|x|^{2}\right)^{-1} e^{|x|} \exp \left(i e^{|x|}\right)$ are typical examples for the aove $q_{1}(x)$.

From the result of the previous section, we already know that the multiplication operator $u \mapsto\left(q_{1}(x)+q_{2}(x)\right) u$ is bounded from $H^{1}$ to $H^{-1}$. We consider its further property under the stricter assumption of this section.

Lemma 7. For any $u \in H^{1}, v \in C_{0}^{\infty}$ and any constant $R \geq 1$, the following holds.

$$
\left|\int_{|x| \geq R} q_{1}(x) u(x) \overline{v(x)} d x\right| \leq \varepsilon(R)(\|u\|\|\nabla v \mid+\| \nabla u\|\| v \|)+(N-1) \varepsilon(R)\|u\|\|v\| .
$$

Proof. We may assume $u \in H^{1} \cap C^{\infty}$. Notice that $v \in C_{0}^{\infty}$.

$$
\begin{aligned}
\int_{|x| \geq R} q_{1}(x) u(x) \overline{v(x)} d x & =\int_{S^{N-1}} \int_{R}^{\infty}\left\{\frac{\partial}{\partial r}\left(Q_{1}(r \omega)-Q_{1}(R \omega)\right)\right\} r^{N-1} u(r \omega) \overline{v(r \omega)} d r d \omega \\
& =-\int_{S^{N-1}} \int_{R}^{\infty}\left\{Q_{1}(r \omega)-Q_{1}(R \omega)\right\} \frac{\partial}{\partial r} r^{N-1} u(r \omega) \overline{v(r \omega)} d r d \omega
\end{aligned}
$$

Since $\left|Q_{1}(r \omega)-Q_{1}(R \omega)\right|<\varepsilon(R)$ for $r \geq R \geq 1$, 


$$
\begin{aligned}
& \left|\int_{|x| \geq R} q_{1}(x) u(x) \overline{v(x)} d x\right| \\
& \leq \varepsilon(R) \int_{S^{N-1}} \int_{R}^{\infty} r^{N-1}\left\{(N-1) r^{-1}|u(r \omega)||v(r \omega)|\right. \\
& \quad+|(\partial / \partial r) u(r \omega)||v(r \omega)|+|u(r \omega)||(\partial / \partial r) v(r \omega)|\} d r d \omega \\
& \leq(N-1) R^{-1} \varepsilon(R) \int_{|x| \geq R}|u(x)||v(x)| d x \\
& \quad+\varepsilon(R) \int_{|x| \geq R}(|\nabla u(x)||v(x)|+|u(x)||\nabla v(x)|) d x \\
& \leq(N-1) \varepsilon(R)\|u\|\|v\|+\varepsilon(R)(\|u\|\|\nabla v \mid+\| \nabla u\|\| v \|) .
\end{aligned}
$$

\section{LEMMA 8. The multiplication operator}

$$
u \mapsto\left(1-\chi_{R}(x)\right) q(x) u
$$

defines a bounded map from $H^{1}$ to $H^{-1}$ with norm not larger than $2 N \varepsilon(R)$. Here $\chi_{R}(x)$ is the characteristic function of the open ball $B_{R}=\left\{x \in \mathbf{R}^{N}:|x|<R\right\}$.

ProOF. Since $\left|q_{2}(r \omega)\right|<\varepsilon(R)$ for $r \geq R$, we have

$$
\left|\int_{|x| \geq R} q_{2}(x) u(x) \overline{v(x)} d x\right| \leq \varepsilon(R)\|u\|\|v\|
$$

for $u, v \in H^{1}$. Using this inequality and the previous lemma,

$$
\begin{aligned}
\left|\int_{\mathbf{R}^{N}}\left(1-\chi_{R}(x)\right) q(x) u(x) \overline{v(x)} d x\right| & =\left|\int_{|x| \geq R}\left(q_{1}(x)+q_{2}(x)\right) u(x) \overline{v(x)} d x\right| \\
& \leq N \varepsilon(R)\|u\|\|v\|+\varepsilon(R)(\|u\|\|\nabla v \mid+\| \nabla u\|\| v \|) \\
& \leq(N+1) \varepsilon(R)\left(\|u\|^{2}+\|\left.\nabla u\right|^{2}\right)^{1 / 2}\left(\|v\|^{2}+\|\nabla v\|\right)^{1 / 2} .
\end{aligned}
$$

This implies the claim.

Proposition 9. Let $\left\{u_{n}\right\} \subset H^{1}$ be an arbitrary bounded sequence. Then $\left\{q(x) u_{n}(x)\right\} \subset H^{-1}$ has a converging subsequence.

REMARK. In other words, the multiplication operator $u \mapsto q(x) u$ from $H^{1}$ to $H^{-1}$ is compact. However, it is generally unbounded as a map from $H^{2}$ to $L^{2}$. 
(e.g., $\left.q(x)=|x|^{3} \sin \left(|x|^{5}\right), u(x)=\left(1+|x|^{2}\right)^{-(N+1) / 4}\right)$. That is, it may be relatively unbounded with respect to $(-\triangle)$ in the usual framework.

Proof. The Rellich theorem and $q(x) \in L_{l o c}^{\infty}$ imply $u \mapsto \chi_{j}(x) q(x) u$ is a compact operator from $H^{1}$ to $L^{2} \subseteq H^{-1}$ for each $j=1,2, \ldots$

Let us choose $R=1,2, \ldots, j, \ldots$ in Lemma 8 . Then we have

$$
\left\|\left(1-\chi_{j}(x)\right) q(x) u_{n}\right\|_{H^{-1}} \leq 2 N \varepsilon(j)\left\|u_{n}\right\|, \quad \lim _{j \rightarrow \infty} 2 N \varepsilon(j)=0 .
$$

Let us assume from now on that $\left\|u_{n}\right\|_{H^{1}} \leq 1$ for simplicity.

By the compactness of $u \mapsto \chi_{1}(x) q(x) u$, we can choose a subsequence $\left\{u_{j}^{(1)}\right\}_{j}$ of $\left\{u_{n}\right\}_{n}$ such that $\chi_{1}(x) q(x) u_{j}^{(1)}$ converges in $L^{2} \subset H^{-1}$ and

$$
\begin{aligned}
\limsup _{j, k \rightarrow \infty}\left\|q(x) u_{j}^{(1)}-q(x) u_{k}^{(1)}\right\|_{H^{-1}} & \leq \limsup _{j, k \rightarrow \infty}\left\|\left(1-\chi_{1}(x)\right)\left(q(x) u_{j}^{(1)}-q(x) u_{k}^{(1)}\right)\right\|_{H^{-1}} \\
& \leq 4 N \varepsilon(1) .
\end{aligned}
$$

In the same way, we choose a subsequence $\left\{u_{j}^{(2)}\right\}_{j}$ of $\left\{u_{j}^{(1)}\right\}_{j}$ such that $\chi_{2}(x) q(x) u_{j}^{(2)}$ converges in $L^{2} \subset H^{-1}$ and

$$
\limsup _{j, k \rightarrow \infty}\left\|q(x) u_{j}^{(2)}-q(x) u_{k}^{(2)}\right\|_{H^{-1}} \leq 4 N \varepsilon(2) .
$$

Repeating the same procedure, we finally have $\left\{u_{j}^{(\ell)}\right\}(\ell, j=1,2, \ldots)$ such that

$$
\limsup _{j, k \rightarrow \infty}\left\|q(x) u_{j}^{(\ell)}-q(x) u_{k}^{(\ell)}\right\|_{H^{-1}} \leq 4 N \varepsilon(\ell) .
$$

Using the diagonal process, we have

$$
\limsup _{j, k \rightarrow \infty}\left\|q(x) u_{j}^{(j)}-q(x) u_{k}^{(k)}\right\|_{H^{-1}}=0
$$

since $\lim _{j \rightarrow \infty} 4 N \varepsilon(j)=0$. In other words, the subsequence $\left\{q(x) u_{j}^{(j)}\right\}$ converges in $H^{-1}$.

THEOREM 10. Let

$$
T_{0} u=-\triangle u+q(x) u, \quad \operatorname{Dom}\left(T_{0}\right)=C_{0}^{\infty}\left(\mathbf{R}^{N}\right) .
$$

Assume

$$
\begin{gathered}
q(x)=q_{1}(x)+q_{2}(x), \quad q_{1}(x) \in L_{l o c}^{\infty}\left(\mathbf{R}^{N}\right), \quad q_{2}(x) \in L^{\infty}\left(\mathbf{R}^{N}\right), \\
\lim _{r_{1}, r_{2} \rightarrow \infty} \sup _{\omega \in S^{N-1}}\left|\int_{r_{1}}^{r_{2}} q_{1}(\rho \omega) d \rho\right|=0
\end{gathered}
$$


and

$$
\lim _{r \rightarrow \infty} \sup _{\omega \in S^{N-1}}\left|q_{2}(r \omega)\right|=0 .
$$

Then the Friedrichs extension $T$ of $T_{0}$ satisfies

$$
\sigma_{e s s}(T)=[0, \infty)
$$

Proof. Let $\mu>0$ be sufficiently large. Then $-\mu \in \rho(T) \cap \rho(-\triangle)$ holds and $T+\mu,-\triangle+\mu$ are isomorphic maps from $H^{1}$ to $H^{-1}$. (Strictly speaking, consider both of closed sectorial forms with domain $H^{1}$ which are associated with $T+\mu$ and $-\triangle+\mu$.)

Let us prove $(T+\mu)^{-1}-(-\triangle+\mu)^{-1}$ is a compact operator in $L^{2}$. Suppose that $\left\{u_{n}\right\} \subset L^{2}$ is an arbitrary bounded sequence. Note that

$$
\begin{aligned}
(T+\mu)^{-1}-(-\Delta+\mu)^{-1} & \\
& =(T+\mu)^{-1}(-\Delta+\mu)(-\triangle+\mu)^{-1}-(T+\mu)^{-1}(T+\mu)(-\Delta+\mu)^{-1} \\
& =(T+\mu)^{-1}\{-q(x)\}(-\triangle+\mu)^{-1} .
\end{aligned}
$$

Note also that $\left\{(-\triangle+\mu)^{-1} u_{n}\right\}$ is a bounded sequence in $H^{2} \subset H^{1}$. By Lemma 9, there exists a subsequence $\left\{u_{n_{j}}\right\}$ of $\left\{u_{n}\right\}$ such that $q(x)(-\Delta+\mu)^{-1} u_{n_{j}}$ converges in $H^{-1}$, hence

$$
\left\{(T+\mu)^{-1}-(-\triangle+\mu)^{-1}\right\} u_{n_{j}}=-(T+\mu)^{-1} q(x)(-\triangle+\mu)^{-1} u_{n_{j}}
$$

converges in $H^{1} \subset L^{2}$. Since $\left\{u_{n}\right\} \subset L^{2}$ is an arbitrary bounded sequence in $L^{2}$, this implies $(T+\mu)^{-1}-(-\triangle+\mu)^{-1}$ is a compact operator from $L^{2}$ into itself. Therefore

$$
\sigma_{e s s}(T)=\sigma_{e s s}(-\triangle)=[0, \infty)
$$

REMARK. The present theorem can be extended as follows by the result of F. Gesztesy et al. [1] and a rather lengthy argument, although the minimal operator has not yet been proved essentially m-sectorial.

Let $q_{1}(x), q_{2}(x)$ in $q(x)=q_{1}(x)+q_{2}(x)$ belong to $L_{\text {loc }}^{2}, q_{2}(x)$ be $(-\triangle)$ compact and $\chi_{R}(x) q_{1}(x)$ be $(-\triangle)$-compact for all $R>0$. Let the other assumption be the same. Then the result remain the same. 


\section{Yorimasa OsHIME}

\section{References}

[ 1 ] F. Gesztesy, M. Malamud, M. Mitrea and S. Naboko, Generalized polar decompositions for closed operators in Hilbert spaces and some applications, Integr. Equ. Oper. Theory 64 (2009), 83-113.

[2] T. Kato, Perturbation Theory for Linear Operators, 2nd edition, 1984, Springer.

[ 3 ] V. B. Mateev and M. M. Skriganov, Wave operators for Schrödinger equation with rapidly oscillating potential, Soviet Math. Dokl. 13 (1972), 185-188.

[4] M. Reed and B. Simon, Method of Modern Mathematical Physics, vol. II, "Fourier Analysis, Self-adjointness", Academic Press.

[5] I. Sasaki, Schrödinger operators with rapidly oscillating potentials, Integr. Equ. Oper. Theory 58 (2007), 563-571.

[6] M. M. Skriganov, On the spectrum of the Schrödinger operators with a rapidly oscillating potential, Proc. Steklov Inst. Math. 125 (1973), 177-185.

Doshisha University 